\title{
Interaction of Auditory Status, Child Maltreatment, and Victimization of Intimate Partner Violence
}

\author{
By Judy L. Porter* \\ Laverne Mcquiller Williams ${ }^{\dagger}$ \\ Kristy Love
}

This study explores the effects of being mistreated as a child and the possibility of becoming a victim of intimate partner violence with an emphasis on auditory status. Deaf or head of hearing children experience childhood mistreatment, including psychological and physical abuse at a higher rate than hearing children. This study examines the hypothesis that having been mistreated as a child and being a member of a minority and disabled group leads to a greater likelihood of becoming a victim of interpersonal violence. The study also researches the auditory status of the partner dyad-hearing with hearing, hearing with Deaf, hearing with hard of hearing, Deaf with Deaf, and hard of hearing with hard of hearing. The hypothesis that Deaf or hard of hearing partnered with hearing will experience greater rates of abuse is tested.

Keywords: abuse - psychological, child-maltreatment, deaf, physical.

\section{Introduction}

Intimate partner violence (IPV) is pervasive on college and university campuses. Intimate partner violence refers to violence between individuals in dating and cohabiting relationships and encompasses physical and psychological abuse (World Health Organization 2012). Although several methodological issues - including the time frame assessed, definitions employed, and questions asked-make comparisons difficult, research has consistently shown that college students both within and outside of the United States are at a high risk of intimate partner violence (Chan et al. 2008, Fass et al. 2008, NCADV 2016).

Because IPV among college students occurs at alarmingly high rates, it is important to understand the factors that may increase the risk of victimization. One possible risk factor is child maltreatment. Child maltreatment is one of the most commonly studied risk factors for dating violence (Jennings et al. 2011, Riggs et al. 2009). Both witnessing interparental violence and experiencing child abuse have been found to increase the likelihood of victimization among college students (Langhinrichsen-Rohling et al. 2004).

Despite increased attention paid to IPV on college and university campuses,

\footnotetext{
*Associate Professor, Department of Criminal Justice, Rochester Institute of Technology, USA. ${ }^{\dagger}$ Associate Dean, College of Liberal Arts, Rochester Institute of Technology, USA.

*American Sign Language Interpreter, National Institute for the Deaf, Rochester Institute of Technology, USA.
} 
only a limited number of studies have focused on IPV among college students who are Deaf ${ }^{1}$ or hard of hearing (Anderson 2010, Anderson and Leigh 2011, Mason 2010, Porter et al. 2011a, 2011b). The importance of examining IPV within the context of auditory status cannot be understated as studies based on U.S. community samples have indicated that Deaf and hard of hearing individuals are more likely to be victimized over their lifetime than hearing individuals (Brownridge 2009, Johnston-McCabe et al. 2011). Accordingly, the purpose of this study is to examine the extent of victimization among a sample of female and male college students and whether these experiences vary by auditory status. Moreover, this study examines IPV and the auditory status of the partner dyad - hearing with hearing, hearing with Deaf, hearing with hard of hearing, Deaf with Deaf, and hard of hearing with hard of hearing to investigate whether differing experiences with IPV exist within these dyads. In addition, we also explore whether child maltreatment, namely childhood physical abuse and witnessing interparental abuse, increase the risk of IPV for adult victimization. Having a better understanding of the rates of victimization and risk factors that may increase students' risk of experiencing abuse is important in developing and implementing target prevention and intervention programs.

\section{IPV among College Students}

Estimates suggest that college students experience high rates of dating violence that range from $17 \%$ and $39 \%$ for physical abuse (Dye and Eckhard 2000, Edwards 2015, Orcutt et al. 2005, Perry and Fromuth 2005, Smith et al. 2014 ) and $65 \%$ and $86 \%$ for psychological abuse (Black et al. 2010, Cercone et al. 2005, Cogan and Ballinger 2006, Forke et al. 2008, Hines and Saudino 2003). Both men and women have been found experience abuse while in college. For example, Perry and Fromuth's (2005) study of 50 unmarried college couples from a public southern university found that $30 \%$ of women and $42 \%$ of men reported receiving physical violence by their partner. Other studies suggest that the rates of receiving and inflicting abuse are similar. Similarly, Harned's (2001) study of college men and women reported that both genders experienced similar rates of physical abuse from their partners and both genders were likely to be victims of psychological abuse.

To date only a handful of studies have examined auditory status within the context of IPV in college relationships. Collectively, these studies using college samples in the U.S. indicate that prevalence rates of experiencing intimate partner violence were more than doubled for Deaf and hard of hearing individuals when compared to hearing populations (Anderson and Leigh 2011, Porter and McQuiller Williams 2011a, 2011b). For example, in a 2010 study of Deaf

\footnotetext{
${ }^{1}$ In the United States, Deaf people do not see themselves as having a disability, but rather have a culture and way of communication that is denied by the dominate hearing culture (Sadusky and Obinna 2002). The use of the capital " $\mathrm{D}$ " is to acknowledge the unique cultural identity of Deaf individuals. This includes a strong affiliation to the Deaf community and a shared language (American Sign Language) (Anderson et al. 2011).
} 
women undergraduates at a college in Washington D.C., it was found that Deaf women were twice as likely to report experiencing physical assault and psychological aggression as hearing students in the previous year (Anderson and Leigh 2011). Although these empirical studies contribute to our understanding of the prevalence of IPV victimization among Deaf and hard of hearing college students, little is known the risk factors that increase the risk of victimization. To date, only one study has examined the prevalence of IPV comparing hearing- Deaf versus Deaf -Deaf relationships (Anderson and Pezzarossi 2013). Using a sample of college women, findings revealed that hearing status of one's partner did not account for significant differences in psychological abuse or physical abuse. Although this study is helpful in contributing to our understanding of IPV comparing differing groups by auditory status, the study is limited in that it only focused on the victimization experiences of women.

\section{Child Maltreatment and the Risk of IPV Victimization and Perpetration}

In analyzing risk factors for dating violence, numerous studies using hearing samples have examined the link between experiencing child abuse and/or witnessing interparental violence in the family of origin and later partner victimization (Cyr et al. 2006, Foshee et al. 2008, Gover at al. 2011, Maas et al. 2010). Research in this area suggests that for both men and women, parent-tochild physical abuse is associated with physical partner violence as both victim and perpetrator (Coffeyet al. 1996, Foshee et al. 2004, Gomez 2011, Jankowski et al. 1999, Lavoie et al. 2002, Millett et al. 2013, O'Keefe 2005, Stith et al. 2000).

Studies examining the association between witnessing interparental violence and subsequent violence have produced mixed results some studies finding significance with partner victimization (Brownridge 2006, Cappell and Heiner 1990, Kwong et al. 2003). However, other studies did not find a significant relationship between family of origin variables and subsequent partner violence (Busby et al. 2008, Foshee et al. 2005). While there is evidence that witnessing or experiencing parental violence is a risk factor for adult victimization, not all children exposed to family of origin violence later inflict or experience violence. As such, researchers have suggested that trajectories for IPV may operate differently for women and women. For example, some studies reveal moderate to strong relationships between interparental violence and subsequent partner victimization only for women (Gover et al. 2008), interparental violence and subsequent partner perpetration only for men (Chen and White 2004), parent-tochild abuse and subsequent partner victimization only for women (Chen and White 2004, Stith et al. 2000), and parent-to-child abuse and subsequent partner perpetration only for women. However, Fergusson, Boden, and Horwood's (2006) results reported no significant victimization differences between witnessing violence and the men and women in their sample. 


\section{Current Study}

The current study adds to the IPV literature in two ways. First, we examine the extent of partner violence victimization among a sample of hearing, Deaf, and hard of hearing male and female college students, and whether these experiences vary by auditory status. By including male and female college students in the analysis, this study is able to explore whether auditory status increases the risk for partner violence for both sexes, not just women. Moreover, this study goes one step further by investigating auditory status of the partner dyad to examine whether IPV experiences differ within these dyads. Second, this study makes a contribution by exploring whether risk factors other than disability increase the risk of partner violence victimization. This is an important contribution because very little, if anything, has been published about risk factors for partner violence, other than disability, for college students who are Deaf or hard of hearing.

\section{Data Collection and Measures}

The purpose of this study was to determine whether data collected indicated significant correlations between auditory status and intimate partner victimization among Deaf or hard of hearing students. The purpose was also to examine if Deaf and hard of hearing students experience and witness family abuse at higher rates than their hearing peers and if that relationship impacts their experiences with partner abuse in college. The cross-sectional data for this study was collected from a northeastern university in the U.S. Thirty-six classes were randomly selected by the researchers. After receiving approval from the Institutional Review Board (IRB), surveys were distributed within the randomly selected classes to all students. Students were informed that the survey was voluntary and they could stop at any time. The survey was distributed in the spring of 2011. A total of 260 respondents completed the survey and we had a response rate of $96 \%$.

\section{Measures}

Gender, auditory status, race and college year status are the independent variables. Three childhood maltreatment variables were assessed: experiencing child abuse, witnessing mother-to-father physical violence and witnessing father-to-mother physical violence. The child abuse measure was created from six items from the Parent-Child Conflict Tactics Scale (Straus et al. 1998) to indicate whether a respondent experienced physical abuse at the hands of a parent, caregiver, or guardian. Witnessing inter-parental abuse was measured by asking respondents whether before the age of 18, they had witnessed their mother hit their father and/or witnessed their father hit their mother. Students were able to answer never, once or twice, three to ten times, or more than ten times. The responses were coded as: $1=$ never, $2=$ once or twice, $3=$ three to ten times and $4=$ more than ten times. Due to sparse responses in the categories 
responses were coded for analysis as dichotomous yes or no.

The child maltreatment variable was created by combining the frequencies from the self-reported questions: "Parent/caregiver threated you with a gun or a knife", "Parent/caregiver choked you", "Parent/caregiver beat you up", and "Parent/ caregiver forced you to have sex (vaginal, anal, or oral intercourse) against your will". Students were able to answer, never, once or twice, three to ten times, or more than ten times. The responses were coded as: $1=$ never, $2=$ once or twice, $3=$ three to ten times and $4=$ more than ten times. Responses were coded dichotomous yes or no due to sparse responses in the categories.

Gender was self-reported and coded as a dichotomous variable where 1= female and $0=$ male. Auditory status was self-reported with the question: "Which best describes your auditory status?" Students were able to answer, hearing, hard of hearing, or Deaf. Race was self-reported with the question: "How do you usually describe yourself?" Race was combined to create a dichotomous variable where $0=$ non-white and $1=$ white.

Conflict Tactics Scale Psychological Index (CTSPsychIndex), Conflict Tactics Scale Physical Index (CTSPhysIndex). To arrive at the measures for psychological and physical abuse within the CTSPsychIndex and CTSPhysIndex, Straus et al.'s (1996) Revised Conflict Tactics Scale (CTS2) was used to measure intimate partner violence by "a partner" over the previous school year. Use of the term "partner" denotes intimate partner violence may exist among heterosexual and same-sex partners. The CTS2 is a commonly used measure of intimate partner violence that measures the frequency with which respondents had experienced psychological and physical abuse from their dating partners. Three items assessed psychological abuse (e.g., insults, and threats) and seven items assessed physical abuse (e.g., slapping, pushing, kicking). Psychometric analyses conducted by Anderson and Leigh (2011) reported sound construct validity between the psychological and physical abuse scales for Deaf and Hard of Hearing college students. CTSPsychIndex was created by combining the self-reported questions: "Partner insulted or swore at you?", "Partner put you down in front of family and/or friends?", and "Partner threatened to hit or throw something at you?" CTSPhysIndex was created by combining the selfreported questions: "Partner pushed, grabbed, or shoved you?", "Partner slapped you?", "Partner kicked or bit you?", "Partner beat you up?", "Partner hit you or tried to hit you with something?", "Partner choked you?", "Partner threatened you with a gun or a knife?"

\section{Findings/Results}

\section{Demographic Profile of Respondents}

Women were slightly over half of the respondents (54.6\%). The majority of respondents were white (55.8\%), black not Hispanic were nearly $16 \%$ of the respondents, while Hispanic or Latino were 13\%, Asian or Pacific Islander were just under 10\%, with America Indian or Alaskan Native at under 3\% of 
the respondents. Auditory status was self-identified and characterized by hearing, Deaf, and hard of hearing. About $45 \%$ of respondents identified as hearing, close to $40 \%$ identified as Deaf, and about $15 \%$ identified as hard of hearing (Table 1).

Table 1. Respondents' Gender, Race/Ethnicity, and Auditory Status (N=260)

\begin{tabular}{|l|c|c|}
\hline & n & Percent \\
\hline Gender & & \\
\hline Male & 117 & 45.0 \\
\hline Female & 142 & 54.6 \\
\hline Transgender & 1 & .4 \\
\hline Total & 260 & 100.0 \\
\hline Race/Ethnicity & & \\
\hline White & 145 & 55.8 \\
\hline Black not Hispanic & 41 & 15.8 \\
\hline Hispanic/Latino & 34 & 13.1 \\
\hline Asian/Pacific Islander & 25 & 9.6 \\
\hline American Indian/Alaskan Native & 7 & 2.7 \\
\hline Other & 7 & 2.7 \\
\hline Missing & 1 & .4 \\
\hline Total & 260 & 100.0 \\
\hline Auditory Status & & \\
\hline Hearing & 118 & 46.1 \\
\hline Deaf & 98 & 38.3 \\
\hline Hard of Hearing & 40 & 15.6 \\
\hline Total & 256 & 100.0 \\
\hline Missing & 4 & \\
\hline Total & 260 & \\
\hline
\end{tabular}

Of the 255 respondents 138 were Deaf or hard of hearing (DHH) compared with 117 respondents who identified as hearing. The majority of respondents identified as white $(n=103)$ compared with those who identified as a member of a racial or ethnic minority group $(n=85)$. Of the 47 hearing men 31 were white and 16 were members of a racial or ethnic minority (REM). Of the 70 hearing women 38 were white and 32 were REM. Among the $138 \mathrm{DHH}$ respondents, 67 were men and 71 were women. There were 38 white hearing women and 31 white hearing men compared to 16 REM hearing men and 32 REM hearing women. There were 38 DHH white men and 29 REM DHH men with 34 white DHH women and 37 REM DHH women (Table 2). 
Table 2. Cross-tabulation of Gender by Race/Ethnicity by Auditory Status $(N=260)$

\begin{tabular}{|c|c|c|c|c|c|c|}
\hline \multicolumn{7}{|c|}{ Cross-tabulation of Gender by Race/Ethnicity by Auditory Status } \\
\hline \multirow{2}{*}{\multicolumn{4}{|c|}{ Auditory Status }} & \multicolumn{2}{|c|}{ Race/Ethnicity } & \multirow{3}{*}{$\begin{array}{c}\text { Total } \\
47\end{array}$} \\
\hline & & & & White & $\mathbf{R E M}^{2}$ & \\
\hline \multirow{9}{*}{ Hearing } & \multirow{6}{*}{ Gender } & \multirow{3}{*}{ Male } & Count & 31 & 16 & \\
\hline & & & $\begin{array}{l}\% \text { within respondent } \\
\text { gender }\end{array}$ & $66.0 \%$ & $34.0 \%$ & $100.0 \%$ \\
\hline & & & $\%$ within respondent race & $44.9 \%$ & $33.3 \%$ & $40.2 \%$ \\
\hline & & \multirow{3}{*}{ Female } & Count & 38 & 32 & 70 \\
\hline & & & $\begin{array}{l}\% \text { within respondent } \\
\text { gender }\end{array}$ & $54.3 \%$ & $45.7 \%$ & $100.0 \%$ \\
\hline & & & $\%$ within respondent race & $55.1 \%$ & $66.7 \%$ & $59.8 \%$ \\
\hline & \multirow{3}{*}{\multicolumn{2}{|c|}{ Total }} & Count & 69 & 48 & 117 \\
\hline & & & $\begin{array}{l}\% \text { within respondent } \\
\text { gender }\end{array}$ & $59.0 \%$ & $41.0 \%$ & $100.0 \%$ \\
\hline & & & $\%$ within respondent race & $100.0 \%$ & $100.0 \%$ & $100.0 \%$ \\
\hline \multirow{9}{*}{$\mathrm{DHH}^{3}$} & \multirow{6}{*}{ Gender } & \multirow{3}{*}{ male } & Count & 38 & 29 & 67 \\
\hline & & & $\begin{array}{l}\text { \% within respondent } \\
\text { gender }\end{array}$ & $56.7 \%$ & $43.3 \%$ & $100.0 \%$ \\
\hline & & & $\%$ within respondent race & $52.8 \%$ & $43.9 \%$ & $48.6 \%$ \\
\hline & & \multirow{3}{*}{ female } & Count & 34 & 37 & 71 \\
\hline & & & $\begin{array}{l}\% \text { within respondent } \\
\text { gender }\end{array}$ & $47.9 \%$ & $52.1 \%$ & $100.0 \%$ \\
\hline & & & $\%$ within respondent race & $47.2 \%$ & $56.1 \%$ & $51.4 \%$ \\
\hline & \multirow{3}{*}{\multicolumn{2}{|c|}{ Total }} & Count & 72 & 66 & 138 \\
\hline & & & $\begin{array}{l}\% \text { within respondent } \\
\text { gender }\end{array}$ & $52.2 \%$ & $47.8 \%$ & $100.0 \%$ \\
\hline & & & $\%$ within respondent race & $100.0 \%$ & $100.0 \%$ & $100.0 \%$ \\
\hline \multirow{9}{*}{ Total } & \multirow{6}{*}{ Gender } & \multirow{3}{*}{ male } & Count & 69 & 45 & 114 \\
\hline & & & $\begin{array}{l}\text { \% within respondent } \\
\text { gender }\end{array}$ & $60.5 \%$ & $39.5 \%$ & $100.0 \%$ \\
\hline & & & $\%$ within respondent race & $48.9 \%$ & $39.5 \%$ & $44.7 \%$ \\
\hline & & \multirow{3}{*}{ female } & Count & 72 & 69 & 141 \\
\hline & & & $\begin{array}{l}\% \text { within respondent } \\
\text { gender }\end{array}$ & $51.1 \%$ & $48.9 \%$ & $100.0 \%$ \\
\hline & & & $\%$ within respondent race & $51.1 \%$ & $60.5 \%$ & $55.3 \%$ \\
\hline & \multirow{3}{*}{\multicolumn{2}{|c|}{ Total }} & Count & 141 & 114 & 255 \\
\hline & & & $\begin{array}{l}\% \text { within respondent } \\
\text { gender }\end{array}$ & $55.3 \%$ & $44.7 \%$ & $100.0 \%$ \\
\hline & & & $\%$ within respondent race & $100.0 \%$ & $100.0 \%$ & $100.0 \%$ \\
\hline
\end{tabular}

Experiences of Psychological and Physical Abuse

Nearly $60 \%$ of respondents reported experiencing psychological or physical abuse $(\mathrm{n}=145,59.2 \%)$ (Table 3 ). Over $40 \%$ of responders reported physical abuse (Table 4), while over half reported psychological abuse (Table 5). There

\footnotetext{
${ }^{2} \mathrm{REM}$ indicates a member of a racial or ethnic minority

${ }^{3} \mathrm{DHH}$ indicates a respondent who identified as Deaf of hard of hearing
} 
was not statistical significance for any of the auditory statuses for either a combined physical/psychological or separate physical and psychological abuse (Tables 6-8). Over $60 \%$ of the offenders were a partner of the respondent at the time of the abuse $(n=105,60.7 \%)$. The next largest category of offenders were described as a date $(\mathrm{n}=37,21.4 \%)$ (Table 9$)$.

Table 3. Psychological and Physical Abuse for All Respondents $(N=260)$

\begin{tabular}{|l|c|c|c|}
\hline & Frequency & Percent & Cumulative Percent \\
\hline No & 100 & 40.8 & 40.8 \\
\hline Yes & 145 & 59.2 & 100 \\
\hline Total & 245 & 100.0 & \\
\hline Missing & 15 & & \\
\hline Total & 260 & & \\
\hline
\end{tabular}

Table 4. Physical Abuse for all Respondents $(N=260)$

\begin{tabular}{|l|c|c|c|}
\hline & Frequency & Percent & Cumulative Percent \\
\hline No & 141 & 57.3 & 57.3 \\
\hline Yes & 105 & 42.7 & 100 \\
\hline Total & 246 & 100.0 & \\
\hline Missing & 14 & & \\
\hline
\end{tabular}

Table 5. Psychological Abuse for all Respondents $(N=260)$

\begin{tabular}{|l|c|c|c|}
\hline & Frequency & Percent & Cumulative Percent \\
\hline No & 118 & 47.4 & 47.4 \\
\hline Yes & 131 & 52.6 & 100 \\
\hline Total & 249 & 100 & \\
\hline Missing & 11 & & \\
\hline Total & 260 & & \\
\hline
\end{tabular}

Table 6. Psychological and Physical Abuse for All Respondents by Auditory Status $(N=245)$

\begin{tabular}{|c|c|c|c|c|}
\hline & \multicolumn{3}{|c|}{$\begin{array}{c}\text { Physical and Psychological } \\
\text { Abuse }\end{array}$} & \multirow{2}{*}{$\begin{array}{c}\begin{array}{c}\text { Chi-Square } \\
\text { (2-sided) }\end{array} \\
307\end{array}$} \\
\hline & No & Yes & Total & \\
\hline $\begin{array}{l}\text { Hearing } \\
\% \text { within auditory status } \\
\% \text { within psychological } \\
\text { abuse }\end{array}$ & $\begin{array}{c}47 \\
41.6 \\
47.0\end{array}$ & $\begin{array}{c}66 \\
58.4 \\
45.5\end{array}$ & $\begin{array}{c}113 \\
100 \\
436.1\end{array}$ & \\
\hline $\begin{array}{l}\text { Deaf } \\
\% \text { within auditory status } \\
\% \text { within psychological } \\
\text { abuse }\end{array}$ & $\begin{array}{c}42 \\
44.2 \\
42.0\end{array}$ & $\begin{array}{c}53 \\
55.8 \\
36.6\end{array}$ & $\begin{array}{c}95 \\
100 \\
38.8\end{array}$ & \\
\hline $\begin{array}{l}\text { Hard of Hearing } \\
\% \text { within auditory status } \\
\% \text { within psychological } \\
\text { abuse }\end{array}$ & $\begin{array}{c}11 \\
29.7 \\
11.0\end{array}$ & $\begin{array}{c}26 \\
70.3 \\
17.9\end{array}$ & $\begin{array}{c}37 \\
100 \\
15.1\end{array}$ & \\
\hline Total & 100 & 145 & 245 & \\
\hline
\end{tabular}


Table 7. Physical Abuse for All Respondents by Auditory Status $(N=245)$

\begin{tabular}{|l|c|c|c|c|}
\hline & \multicolumn{3}{|c|}{ Physical and Psychological Abuse } & $\begin{array}{c}\text { Chi-Square } \\
\text { (2-sided) }\end{array}$ \\
\hline & No & Yes & Total & .795 \\
\hline Hearing & 63 & 50 & 113 & \\
\% within auditory status & 55.8 & 44.2 & 100 & \\
\% within psychological abuse & 44.7 & 47.6 & 45.9 & \\
\hline Deaf & 57 & 38 & 95 & \\
\% within auditory status & 60.0 & 40.0 & 100 & \\
\% within psychological abuse & 40.4 & 36.2 & 38.6 & \\
\hline Hard of Hearing & 21 & 17 & 38 & \\
\% within auditory status & 55.3 & 44.7 & 100 & \\
\% within psychological abuse & 14.9 & 16.2 & 15.4 & \\
\hline Total & 141 & 105 & 246 & \\
\hline
\end{tabular}

Table 8. Psychological Abuse for All Respondents by Auditory Status $(N=245)$

\begin{tabular}{|c|c|c|c|c|}
\hline & \multicolumn{3}{|c|}{$\begin{array}{c}\text { Physical and Psychological } \\
\text { Abuse }\end{array}$} & $\begin{array}{l}\text { Chi-Square } \\
\text { (2-sided) }\end{array}$ \\
\hline & No & Yes & Total & .639 \\
\hline $\begin{array}{l}\text { Hearing } \\
\% \text { within auditory status } \\
\% \text { within psychological } \\
\text { abuse }\end{array}$ & $\begin{array}{c}54 \\
47.4 \\
45.8\end{array}$ & $\begin{array}{c}60 \\
52.6 \\
45.8\end{array}$ & $\begin{array}{c}114 \\
100 \\
45.8\end{array}$ & \\
\hline $\begin{array}{l}\text { Deaf } \\
\% \text { within auditory status } \\
\% \text { within psychological } \\
\text { abuse }\end{array}$ & $\begin{array}{c}48 \\
50.0 \\
40.7\end{array}$ & $\begin{array}{c}48 \\
50.0 \\
36.6\end{array}$ & $\begin{array}{c}96 \\
100 \\
38.6\end{array}$ & \\
\hline $\begin{array}{l}\text { Hard of Hearing } \\
\% \text { within auditory status } \\
\% \text { within psychological } \\
\text { abuse }\end{array}$ & $\begin{array}{c}16 \\
41.0 \\
13.6\end{array}$ & $\begin{array}{c}23 \\
59.0 \\
17.6\end{array}$ & $\begin{array}{c}39 \\
100 \\
15.7\end{array}$ & \\
\hline Total & 118 & 131 & 249 & \\
\hline
\end{tabular}

Table 9. Relationship with Abuser

\begin{tabular}{|l|c|c|c|}
\hline & Frequency & Percent & Cumulative Percent \\
\hline Partner & 105 & 60.7 & 60.7 \\
\hline Spouse & 3 & 1.7 & 62.4 \\
\hline Acquaintance & 7 & 4.0 & 66.5 \\
\hline Date & 37 & 21.4 & 87.9 \\
\hline Stranger & 9 & 5.2 & 93.1 \\
\hline Family member & 4 & 2.3 & 95.4 \\
\hline Other & 8 & 4.6 & 100.0 \\
\hline Total & 173 & 100.0 & \\
\hline Missing & 87 & & \\
\hline Total & 260 & & \\
\hline
\end{tabular}

A cross tabulation of victim's and offender's auditory status reveals that the majority of abuse is intra-auditory status. Auditory status achieved statistical significance (Chi-Square .000) - there is a statistically significant difference 
between hearing, Deaf, and hard of hearing victims and offenders. Hearing victims are assaulted by hearing offenders over $75 \%$ of the time, while there are no Deaf offenders with hearing victims and only 2 hearing victims report hard of hearing offenders (20\%). Deaf victims are assaulted $77 \%$ of the time by Deaf offenders but also experience abuse at the hands of hearing offenders $11.3 \%$ of the time. Hard of hearing victims experience abuse at the hands of other hard of hearing individuals $40 \%$ of the time, Deaf offenders $40 \%$ of the time and at the hands of hearing offenders $20 \%$ of the time. Abuse is primarily intra-auditory status for Deaf and Hearing, but not for hard of hearing. Hard of hearing is spread across auditory status although the majority of hard of hearing victims experience abuse at the hands of someone who is either Deaf or hard of hearing, while $20 \%$ of hard of hearing victims experience abuse at the hands of hearing offenders (Table 10).

Table 10. Cross Tabulation of Victims' and Offenders' Auditory Status

\begin{tabular}{|c|c|c|c|c|c|c|c|}
\hline & \multicolumn{3}{|c|}{$\begin{array}{c}\text { Respondent Auditory } \\
\text { Status } \\
\end{array}$} & \multirow[b]{2}{*}{ Total } & \multirow{2}{*}{$\begin{array}{c}\begin{array}{c}\text { Chi- } \\
\text { Square }\end{array} \\
(2- \\
\text { sided })\end{array}$} \\
\hline & & & Hearing & Deaf & $\begin{array}{l}\text { Hard of } \\
\text { Hearing }\end{array}$ & & \\
\hline \multirow{6}{*}{$\begin{array}{l}\text { Offender's } \\
\text { auditory } \\
\text { status }\end{array}$} & \multirow[b]{2}{*}{ Hearing } & Count & 73 & 11 & 13 & 97 & .000 \\
\hline & & $\begin{array}{l}\text { Percent } \\
\text { within } \\
\text { offenders' } \\
\text { auditory } \\
\text { status }\end{array}$ & $75.3 \%$ & $11.3 \%$ & $13.4 \%$ & $100.0 \%$ & \\
\hline & \multirow[b]{2}{*}{ Deaf } & Count & 0 & 47 & 14 & 61 & \\
\hline & & $\begin{array}{l}\text { Percent } \\
\text { within } \\
\text { offenders' } \\
\text { auditory } \\
\text { status }\end{array}$ & $0.0 \%$ & $77.0 \%$ & $23.0 \%$ & $100.0 \%$ & \\
\hline & \multirow[b]{2}{*}{$\begin{array}{l}\text { Hard of } \\
\text { Hearing }\end{array}$} & Count & 2 & 4 & 4 & 10 & \\
\hline & & $\begin{array}{l}\text { Percent } \\
\text { within } \\
\text { offenders' } \\
\text { auditory } \\
\text { status }\end{array}$ & $20.0 \%$ & $40.0 \%$ & $40.0 \%$ & $100.0 \%$ & \\
\hline \multirow{2}{*}{\multicolumn{2}{|c|}{ Total }} & Count & 75 & 62 & 32 & 169 & \\
\hline & & $\begin{array}{l}\text { Offenders' } \\
\text { auditory } \\
\text { status }\end{array}$ & $44.4 \%$ & $36.7 \%$ & $18.9 \%$ & $100.0 \%$ & \\
\hline
\end{tabular}

Table 11 details whether a child witnessed parental abuse by auditory status. Over $63 \%$ of hearing respondents reported they had witnessed parental abuse as a child, while over $50 \%$ of Deaf and over $57 \%$ of hard of hearing had been a child witness of parental abuse. There was not statistical significance between auditory status and witnessing parental abuse as a child (Chi-Square .175).

Table 12 summarizes the experiences of child maltreatment by auditory status. Among hearing respondents $45.3 \%$ reported being victims of child maltreatment while $24.4 \%$ of Deaf respondents and $34.4 \%$ of hard of hearing 
respondents reported being victims of child maltreatment. Auditory status does achieve statistical significance (Chi-Square .004) indicating there is a difference between hearing, Deaf, and hard of hearing individuals who have experienced child maltreatment.

Table 11. Cross Tabulation of Auditory Status by Witnessing Parental Abuse as a Child

\begin{tabular}{|c|c|c|c|c|c|c|}
\hline & \multicolumn{2}{|c|}{$\begin{array}{l}\text { Child Witness } \\
\text { Parental Abuse }\end{array}$} & \multirow[t]{2}{*}{ Total } & \multirow{2}{*}{$\begin{array}{c}\text { Chi- } \\
\text { Square }\end{array}$} \\
\hline & & & No & Yes & & \\
\hline \multirow{6}{*}{$\begin{array}{l}\text { Respondent } \\
\text { Auditory Status }\end{array}$} & \multirow[b]{2}{*}{ Hearing } & Count & 42 & 73 & 115 & .175 \\
\hline & & $\begin{array}{l}\text { \% Within } \\
\text { Respondent } \\
\text { Auditory Status }\end{array}$ & $36.5 \%$ & $63.5 \%$ & $100.0 \%$ & \\
\hline & \multirow[b]{2}{*}{ Deaf } & Count & 45 & 46 & 91 & \\
\hline & & $\begin{array}{l}\text { \% Within } \\
\text { Respondent } \\
\text { Auditory Status }\end{array}$ & $49.5 \%$ & $50.5 \%$ & $100.0 \%$ & \\
\hline & \multirow[b]{2}{*}{$\begin{array}{l}\text { Hard of } \\
\text { Hearing }\end{array}$} & Count & 17 & 23 & 40 & \\
\hline & & $\begin{array}{l}\text { \% Within } \\
\text { Respondent } \\
\text { Auditory Status }\end{array}$ & $42.5 \%$ & $57.5 \%$ & $100.0 \%$ & \\
\hline \multirow{2}{*}{\multicolumn{2}{|c|}{ Total }} & Count & 104 & 142 & 246 & \\
\hline & & $\begin{array}{l}\text { \% Within } \\
\text { Respondent } \\
\text { Auditory Status }\end{array}$ & $42.3 \%$ & $57.7 \%$ & $100.0 \%$ & \\
\hline
\end{tabular}

Table 12. Auditory Status by Child Maltreatment

\begin{tabular}{|c|c|c|c|c|c|c|}
\hline & & & Malt & $\begin{array}{l}\text { Id } \\
\text { itment }\end{array}$ & & $\begin{array}{c}\text { Chi- } \\
\text { Square }\end{array}$ \\
\hline & & & No & Yes & Total & (2-sided) \\
\hline Respondent & Hearing & Count & 63 & 52 & 115 & .004 \\
\hline Auditory Status & & $\begin{array}{l}\% \text { within } \\
\text { Respondent } \\
\text { Auditory Status }\end{array}$ & $54.8 \%$ & $45.2 \%$ & $100.0 \%$ & \\
\hline & Deaf & Count & 68 & 22 & 90 & \\
\hline & & $\begin{array}{l}\text { \% within } \\
\text { Respondent } \\
\text { Auditory Status }\end{array}$ & $75.6 \%$ & $24.4 \%$ & $100.0 \%$ & \\
\hline & Hard of & Count & 29 & 10 & 39 & \\
\hline & Hearing & $\begin{array}{l}\text { \% within } \\
\text { Respondent } \\
\text { Auditory Status }\end{array}$ & $74.4 \%$ & $25.6 \%$ & $100.0 \%$ & \\
\hline Total & & Count & 160 & 84 & 244 & \\
\hline & & $\begin{array}{l}\% \text { within } \\
\text { Respondent } \\
\text { Auditory Status }\end{array}$ & $65.6 \%$ & $34.4 \%$ & $100.0 \%$ & \\
\hline
\end{tabular}

A binomial regression analysis that included child witness, auditory status, gender, and race found that only witnessing parental abuse as a child was statistically significant for being an adult victim of psychological abuse. Individuals who witnessed parental abuse as a child were over nine times as likely as those who had not witnessed parental abuse to become victims of psychological 
abuse as an adult (Table 13).

Table 13. Binomial Regression Analysis of Auditory Status and Witnessing Parental Abuse as a Child and Becoming a Victim of Psychological Abuse as an Adult

\begin{tabular}{|l|l|c|c|c|c|c|c|}
\hline \multicolumn{2}{|l|}{} & B & S.E. & Wald & df & Sig. & $\operatorname{Exp(B)}$ \\
\hline \multirow{2}{*}{ Child witness } & 2.240 & .303 & 54.587 & 1 & .000 & 9.389 \\
\cline { 2 - 8 } & Constant & -1.179 & .233 & 25.496 & 1 & .000 & .308 \\
\hline
\end{tabular}

a. Variable(s) entered on step 1: child witness, auditory status, gender, and race/ethnicity.

Table 14 details a binomial regression analysis that examines the effects of witnessing parental abuse as a child and becoming a victim of physical abuse as an adult. The findings indicate that being a child witness increasing the odds of experiencing physical abuse as an adult by 4.663 times. The odds are nearly doubled for women and over $2 \frac{1 / 2}{2}$ times as much for a member of a racial or ethnic minority.

Table 14. Binomial Regression Analysis of Auditory Status and Witnessing Parental Abuse as a Child and Becoming a Victim of Physical Abuse as an Adult

\begin{tabular}{|c|c|c|c|c|c|c|c|}
\hline & B & S.E. & Wald & df & Sig. & $\operatorname{Exp}(B)$ \\
\hline \multirow[t]{4}{*}{ Step $2^{\mathrm{a}}$} & Child witness & 1.540 & .309 & 24.746 & 1 & .000 & 4.663 \\
\hline & Gender & .784 & .297 & 6.948 & 1 & .008 & 2.190 \\
\hline & Race/Ethnic & .959 & .295 & 10.595 & 1 & .001 & 2.610 \\
\hline & Constant & -2.126 & .343 & 38.452 & 1 & .000 & .119 \\
\hline
\end{tabular}

a. Variable(s) entered on step 1: child witness, auditory status, gender, and race/ethnicity.

Table 15 illustrates the relationship between being a victim of child maltreatment and becoming an adult victim of psychological abuse. The findings indicate that those who were victims of child maltreatment were 1.857 times as likely to become a victim of psychological abuse and women were 1.695 as likely to become victims of psychological abuse.

Table 15. Binomial Regression Psychological Abuse and Child Maltreatment

\begin{tabular}{|l|l|c|c|c|c|c|c|}
\hline \multicolumn{2}{|l|}{} & B & S.E. & Wald & df & Sig. & $\operatorname{Exp(B)}$ \\
\hline & Gender & .527 & .269 & 3.849 & 1 & .050 & 1.695 \\
\hline Child Maltreatment & .619 & .284 & 4.751 & 1 & .029 & 1.857 \\
\cline { 2 - 7 } & Constant & -.405 & .211 & 3.678 & 1 & .055 & .667 \\
\hline
\end{tabular}

Table 16 details the results of a binomial regression analysis exploring the effects of being a victim of child maltreatment and the chances of becoming adult victims of physical abuse. The findings indicate that the odds of becoming an adult victim of physical abuse are nearly doubled for women, racial and ethnic minorities and those who were victims of child maltreatment. 
Table 16. Binomial Regression Child Maltreatment and Physical Abuse as an Adult

\begin{tabular}{|c|c|c|c|c|c|c|}
\hline & B & S.E. & Wald & df & Sig. & $\operatorname{Exp}(B)$ \\
\hline Gender & .737 & .287 & 6.599 & 1 & .010 & 2.089 \\
\hline Race/Ethnicity & .922 & .281 & 10.721 & 1 & .001 & 2.513 \\
\hline Child Maltreatment & .746 & .294 & 6.414 & 1 & .011 & 2.108 \\
\hline Constant & -1.409 & .267 & 27.760 & 1 & .000 & .244 \\
\hline
\end{tabular}

\section{Discussion}

Overall, the rate of psychological abuse in the current sample was very high. More than $59 \%$ of the sample reported such abuse. This is comparable with prevalence rates of partner victimization reported by college students as reported in previous studies (Black et al. 2010, Harned 2002, Hines and Saudino 2003). The prevalence of physical violence in the current study is also consistent with prevalence rates in previous studies (Black et al. 2010, Cogan and Ballinger 2006, Hines and Saudino 2003).

The first purpose of this study was to examine the extent of intimate partner violence victimization and among a sample of hearing, Deaf, and hard of hearing male and female college students and whether these experiences varied by auditory status. Previous research indicates higher rates of partner violence victimization for Deaf and hard of hearing individuals than those who are hearing (Anderson and Leigh 2011, Porter et al. 2011a, 2011b). These results were not found in the current study. Rather, no significant differences were observed for psychological abuse or physical abuse among Deaf, hard of hearing, and hearing college students. Although there were no significant differences in being abused by auditory status, the dyad for abuse and auditory status indicated that abuse for hearing and Deaf victims is mostly intra-auditory status. However, this does not hold true for hard of hearing victims whereby 60 percent of their perpetrators were either Deaf or hearing. Barrow (2008) suggests that because hard of hearing individuals are often members of both Deaf culture and mainstream (hearing) culture, this places them at a differing risk of abuse than those who are Deaf or hearing. Along these lines, as Anderson et al. (2011) acknowledges, within the dynamics of the relationships that include one hearing partner and a hard of hearing partner, there lays the potential for the hearing partner to abuse their hearing privilege. This may include the perpetrator using their hearing to manipulate the victim (not share with him or her is being said) and/or communicating with police officers and others because they are hearing (Deaf Hope 2006). This suggests the need for more directed research, including qualitative studies, on victimization and factors that contribute to victimization on college campuses.

The second purpose of this study was to examine whether risk factors, in addition to disability, increase the risk of IPV victimization. Compared to hearing students, Deaf and hard of hearing students were significantly more likely to have witnessed parental abuse and experience child abuse than hearing students. 
However, findings did not indicate that auditory status was statistically significant and different for hearing, Deaf, or hard of hearing for witnessing parental abuse or being the victim of child maltreatment and becoming an adult victim of psychological or physical abuse. The current findings are unexpected given the expansive research on the role of witnessing violence in the family of origin and physical child abuse on subsequent partner violence (Coffey et al. 1996, Foshee et al. 2004, Gover et al. 2011, Jankowski et al. 1999, Marshall and Rose 1988 O'Keefe 2005, Simons et al. 1998). This suggests the need to investigate factors beyond those relied upon with hearing college samples to understand the dynamics of partner violence victimization among Deaf and hard of hearing college students.

Although the current study extends our understanding of the correlates of partner victimization, findings should be viewed with caution in light of several limitations. First, data were obtained by self-report. Thus, the possibility of deliberate response distortion must be considered. Second, present findings may not generalize beyond the particular sample. We note our sample consisted of a small number of Deaf and hard of hearing college men and women who may differ from other groups in their experiences of psychological and physical abuse. The study does, however, provide evidence for future comparisons. Third, the cross-sectional design of this investigation does not allow causal inferences to be made as the temporal order of variables. Future research is also needed concerning specific episodes of psychological and physical abuse to learn more about the dynamics of such abuse.

Finally, substantiation of the present findings, which indicate that partner abuse occurs with frequency among hearing, Deaf and hard of hearing college students, and that most traditional risk factors for partner violence among hearing college samples are not significant when examined for Deaf and hard of hearing students, is crucial for the dissemination of educational information. The findings presented here reiterate the need for a continued focus on risk factors for partner violence, both in terms of victimization and to address and prevent further instances of partner violence.

\section{References}

Anderson ML (2010) Prevalence and Predictors of Intimate Partner Violence Victimization in the Deaf Community. Unpublished Doctoral Dissertation, Gallaudet University, Washington, D.C.

Anderson ML, Leigh IW (2011) Intimate Partner Violence against Deaf Female College Students. Violence against Women 17(7): 822-834. http://doi:10.1177/107780121141 2544.

Anderson ML, Leigh IW, Samar VJ (2011) Intimate Partner Violence against Deaf Women: A Review. Aggression and Violent Behavior 16(3): 200-206. http://doi.org/ 10.1016/j.avb.2011.02.006.

Anderson ML, Kobek Pezzarossi CM (2013) Violence against Deaf Women: Effect of Partner Hearing Status. The Journal of Deaf Studies and Deaf Education 19(3): 411421. http://doi:10.1093/deafed/ent053.

Barrow LB (2008) Criminal Victimization of the Deaf. New York, NY: LFB Scholarly Publishing. 
Black DS, Sussman S, Unger JB (2010) A Further Look at the Intergenerational Transmission of Violence: Witnessing Interparental Violence in Emerging Adulthood. Journal of Interpersonal Violence 25(6): 1022-1042. http://doi:10.1177/ 0886260509340539.

Brownridge DA (2006) Partner Violence against Women with Disabilities. Violence against Women 12: 805-822. http://doi:10.1177/107780120629268.

Busby DM, Holman TB, Walker E (2008) Pathways to Relationship Aggression between Adult Partners. Family Relations 57 (January): 72-83. http://doi:10.1111/j.1741-37 29.2007.00484.x.

Cappell C, Heiner RB (1990) The Intergenerational Transmission of Family Aggression. Journal of Family Violence 5(2): 135-152. http://doi.org/10.1007/BF 00978516.

Carr JL, VanDeuse KM (2002) The Relationship between Family of Origin Violence and Dating Violence in College Men. Journal of Interpersonal Violence 17(6): 630-646. http://doi/10.1177/0886260502017006003.

Cercone JJ, Beach SR, Arias I (2005) Gender Symmetry in Dating Intimate Partner Violence: Does Similar Behavior Imply Similar Constructs? Violence and Victims 20(2): 207-218.

Chan KL, Straus MA, Brownridge DA, Tiwari A, Leung WC (2008) Prevalence of Dating Partner Violence and Suicidal Ideation among Male and Female University Students Worldwide. Journal of Midwifery \& Women's Health 53: 529-537. http://doi:10.1016 /j.jmwh.2008.04.016.

Chen PH, White H (2004) Gender Differences in Adolescent and Young Adult Predictors of Later Intimate Partner Violence. Violence against Women 10(11): 1283-1301. http://doi:10.1177/107780120426900.

Coffey P, Leitenberg H, Henning K, Bennett RT, Jankowski MK (1996) Dating violence: The association between methods of coping and women's psychological adjustment. Violence and Victims 11(3): 227-238.

Cogan R, Ballinger BC (2006) Alcohol Problems and the Differentiation of Partner, Stranger, and General Violence. Journal of Interpersonal Violence 21(7), 924-953. http://doi:10.1177/088626050628917.

Cyr M, McDuff P, Wright J (2006) Prevalence and Predictors of Dating Violence among Adolescent Female Victims of Child Sexual Abuse. Journal of Interpersonal Violence 21(8): 1000-1017. http://doi:10.1177/088626050629020.

Deaf Hope (2006) Deaf Power and Control Wheel. Retrieved from https://bit.ly/2N2XLfX.

Dye ML, Eckhardt CI (2000) Anger, Irrational Beliefs, and Dysfunctional Attitudes in Violent Dating Relationships. Violence and Victims 15(3): 337-350.

Edwards KM, Sylaska KM, Barry JE, Moynihan MM, Banyard VL, Cohn ES, Walsh WA, Ward SK (2015) Physical Dating Violence, Sexual Violence, and Unwanted Pursuit Victimization: A Comparison of Incidence Rates among Sexual-minority and Heterosexual College Students. Journal of Interpersonal Violence 30(4): 580-600. http://doi:10.1177/0886260514535260.

Fass DF, Benson RI, Leggett DG (2008) Assessing Prevalence and Awareness of Violent Behaviors in the Intimate Partner Relationships of College Students using Internet Sampling. Journal of College Student Psychotherapy 22(4): 66-75. https://doi.org/ 10.1080/87568220801952248.

Fergusson DM, Boden JM, Horwood LJ (2006) Examining the Intergenerational Transmission of Violence in New Zealand Birth Cohort. Child Abuse \& Neglect 30: 89-108. http://doi.org/10.1016/j.chiabu.2005.10.006.

Forke CM, Myers RK, Catallozzi M, Schwartz DF (2008) Relationship Violence among Women and Male College Undergraduate Students. Archives of Pediatrics 
\& Adolescent Medicine 162(7): 634-641. http://doi:10.1001/archpedi.162.7.634.

Foshee VA, Benefield TS, Ennett ST, Bauman KE, Suchindean C (2004) Longitudinal Predictors of Severe Physical and Sexual Dating Violence Victimization during Adolescence. Preventive Medicine: An International Journal Devoted to Practice and Theory 39(5): 1007-1016. http://doi:10.1016/j.ypmed.2004.04.014.

Foshee VA, Ennett ST, Bauman T, Benefield T, Suchindra C (2005) The Association between Family Violence and Adolescent Dating Violence Onset: Does it vary by Race, Socioeconomic Status, and Family Structure? Journal of Early Adolescence 25(3): 317-344. http://doi:10.1177/027243160527730.

Gomez AM (2011) Testing the Cycle of Violence Hypothesis: Child Abuse and Adolescent Dating Violence as Predictors of Intimate Partner Violence in Adulthood. Youth \& Society 43(1): 171-192. http://doi:10.1177/0044118X09358313.

Gover A, Kaukinen C, Fox KA (2008) The Relationship between Violence in the Family of Origin and Dating Violence among College Students. Journal of Interpersonal Violence 23(12): 1667-1693. http://doi:10.1177/088626050831433.

Gover A, Park M, Tomsich EA, Jennings W (2011) Dating Violence Perpetration and Victimization among South Korean College Students: A Focus on Gender and Childhood Maltreatment. Journal of Interpersonal Violence 26(6): 1232-1263. http:// doi:10.1177/0886260510368161.

Harned MS (2001) Abused Women or Abused Men? An Examination of the Context and Outcomes of Dating Violence. Violence and Victims 16(3): 269-285.

Hines DA, Saudino KJ (2003) Gender Differences in Psychological, Physical, and Sexual Aggression among College Students using the Revised Conflict Tactics Scales. Violence and Victims 18(2): 197-217.

Holt J, Gillespie W (2008) Intergenerational Transmission of Violence, Threatened Egoism, and Reciprocity: A test of multiple Psychosocial Factors Affecting Intimate Partner Violence. American Journal of Criminal Justice 33(2): 252-266. http://doi: 10.1007/s12103-008-9036-0.

Jankowski MK, Leitenberg H, Henning KR, Coffey P (1999) Intergenerational Transmission of Dating Aggression as a Function of Witnessing only Same Sex Parents vs. Opposite Sex Parents vs. both Parents as Perpetrators of Domestic Violence. Journal of Family Violence 14(3): 267-279. http://doi:10.1023/A:10228144 1666610.

Jennings WG, Park M, Tomisich EA, Gover AR, Akers RL (2011) Assessing the Overlap in Dating Violence Perpetration and Victimization among South Korean College Students: The Influence of Social Learning and Self-control. American Journal of Criminal Justice 36(2): 188-206. http://doi:10.1007/s12103-011-9110-x.

Johnston-McCabe P, Levi-Minzi M, Van Hassely VB, Vanderbeek A (2011) Domestic Violence and Clinical Support in a Clinical Sample of Deaf and Hard of Hearing Women. Journal of Family Violence 26(1): 63-69. https://doi.org/10.1007/s10896010-9342-4.

Kwong MJ, Bartholomew K, Henderson AJZ, Trinke SJ (2003) The Intergenerational Transmission of Relationship Violence. Journal of Family Psychology 17(3): 288301. http://doi:10.1037/0893-3200.17.3.288.

Langhinrichsen-Rohling J, Hankla M, Stromberg CD (2004) The Relationship Behavior Networks of Young Adults: A Test of the Intergenerational Transmission of Violence Hypothesis. Journal of Family Violence 19(3): 139-151. http://doi.org/10.1023/B:JO FV.0000028074.35688.4f.

Lavoie F, Herbert M, Tremblay R, Vitaro F, Vezina L, McDuff P (2002) History of Family Dysfunction and Perpetration of Dating Violence by Adolescent Boys: A Longitudinal Study. Journal of Adolescent Health 30(5): 375-383. http://doi.org/10. 
1016/S1054-139X(02)00347-6.

Maas CD, Fleming CB, Herrenkohl TI, Catalano RF (2010) Childhood Predictors of Teen Dating Violence Victimization. Violence and Victims 25(2): 131-149.

Makepeace JM (1987) Social Factors and Victim Offender Differences in Courtship Violence. Family Relations 36(1): 87-91.

Millett L, Kohl P, Jonson-Reid M, Drake B, Petra M (2013) Child Maltreatment Victimization and Subsequent Perpetration of Young Adult Intimate Partner Violence: An Exploration of Mediating Factors. Child Maltreatment 18(2): 71-84. http://doi:10. 1177/107755957348482.

Murrell AR, Christoff KA, Henning KR (2007) Characteristics of Domestic Violence Offenders: Associations with Exposure to Violence. Journal of Family Violence 22(7): 523-532. http://doi:10.1007/s10896-007-9100-4.

O'Keefe M (2005) Teen Dating Violence: A Review of risk Factors and Prevention Efforts. Violence against Women/National Resource Center on Domestic Violence. Retrieved from https://bit.ly/2C3BV7K.

Orcutt HK, Garcia M, Pickett SM (2005) Female-perpetrated Intimate Partner Violence and Romantic Attachment Style in a College Student Sample. Violence and Victims 20(3): 287-302. http://doi.10.1891/088667005780997938.

Perry AR, Fromuth ME (2005) Courtship Violence using Couple Data: Characteristics and Perceptions. Journal of Interpersonal Violence 20(3): 1078-1095. http://doi:10.1177/ 0886260505278106.

Porter JL, McQuiller Williams L (2011a) Auditory Status and Experiences of Abuse among College Students. Violence and Victims 26(6): 788-798. http://doi:10.1891/08 86-6708.26.6.788.

Porter JL, McQuiller Williams L (2011b) Intimate Violence among Underrepresented Groups on a College Campus. Journal of Interpersonal Violence 26(16): 3210-3224. http://doi:10.1177/0886260510393011.

National Coalition against Domestic Violence (NCADV) (2016) National Statistics. Retrieved from http://www.ncadv.org/learn/statistics.

Riggs DS, Caulfield MB, Fair K (2009) Risk of Intimate Partner Violence: Factors associated with Perpetration and Victimization. In PM Kleespies (ed), Behavioral Emergencies: An Evidence-based Resource for Evaluating and Managing Risk of Suicide, Violence, and Victimization, 189-208. Washington, DC: American Psychological Association.

Stith SM, Rosen KH, Middleton KA, Busch AL, Lundeberg K, Carlton RP (2000) The Intergenerational Transmission of Spouse Abuse: A Meta-analysis. Journal of Marriage and Family 62(August): 640-654. http://doi:10.1111/j.1741-3737.2000.00 640.x.

White HR, Widom CS (2003) Does Childhood Victimization Increase the Risk of Early Death? A 25-year Prospective Study. Child Abuse \& Neglect 27(7): 841-853. http:// dx. doi.org/10.1016/S0145-2134(03)00110-8.

World Health Organization (2012) Global Campaign for Violence Prevention. Violence Prevention Alliance. Retrieved from https://bit.ly/2MHgK00. 
